\title{
Unilateral hypoglossal nerve palsy after the use of a novel supraglottic airway device
}

\author{
R Leong ${ }^{1}$, Quek KH² \\ ${ }^{1}$ Resident, Singhealth Anaesthesia Residency Program, Changi General Hospital, Singapore \\ ${ }^{2}$ Consultant, Department of Anaesthesia and Intensive Care, Changi General Hospital, Singapore
}

\begin{abstract}
Background: The LMA® Protector ${ }^{\mathrm{TM}}$ Airway is a new single-use supraglottic airway device (SAD) with Cuff Pilot ${ }^{\text {TM }}$ Technology that enables visual monitoring of intra-cuff pressure, in order to reduce the risk of complications from an overinflated device. We present a case of unilateral hypoglossal nerve injury despite its use.
\end{abstract}

Case Report: A 39-year old female (ASA I, 52kg, BMI 22) presented for elective left knee arthroscopy and medial meniscus repair. Pre-operative airway assessment was normal. After an uneventful induction of GA, without bag mask ventilation, a size 3 LMA Protector ${ }^{\mathrm{TM}}$ was inserted on first pass and the cuff inflated. The cuff pressure indicator was monitored intra-operatively and remained within the recommended green zone throughout the hour-long operation, with the patient breathing spontaneously on a Sevoflurane/O2/air mixture. Blood pressure was maintained within $20 \%$ of baseline.

The LMA was removed by a registered nurse in the anaesthetic recovery unit (PACU) when the patient was awake. She did not have any complaints while in the PACU but complained of sore throat and dysarthria on the first postoperative day (POD). There was tongue deviation to the right on active protrusion, but no dysgeusia or other neurological signs. A provisional diagnosis of right hypoglossal nerve injury secondary to the SAD was made. By POD7, she reported symptomatic improvement, with normal clinical and nasoendoscopic findings. There was complete resolution of symptoms 3 weeks after surgery with conservative management.

Discussion: Hypoglossal nerve injury is a rare but distressing complication of LMA use. The hypoglossal nerve originates in the medulla oblongata and descends between the internal carotid artery and internal jugular vein after leaving the skull. It courses superficially after the angle of the mandible, passes just above the greater horn of the hyoid bone and enters the floor of the mouth to supply the ipsilateral muscles of the tongue. The greater horn of the hyoid bone is a potential site of injury, as the cuff of the LMA may compress the nerve against bone.
The LMA $®$ Protector $^{\mathrm{TM}}$ Airway is a new single-use supraglottic airway device (SAD) made of silicone and with a preformed curvature for ease of insertion. It has dual drain channels and Cuff Pilot ${ }^{\mathrm{TM}}$ Technology that enables continuous visual monitoring of intra-cuff pressure.

In our patient, possible contributing factors to hypoglossal nerve injury included cuff over-inflation with/without failure of the Cuff Pilot $^{\mathrm{TM}}$ technology, inappropriate sizing and inappropriate placement of an unfamiliar device. Fortuitously, injury is often neuropraxia with complete recovery in 6 months following conservative management.

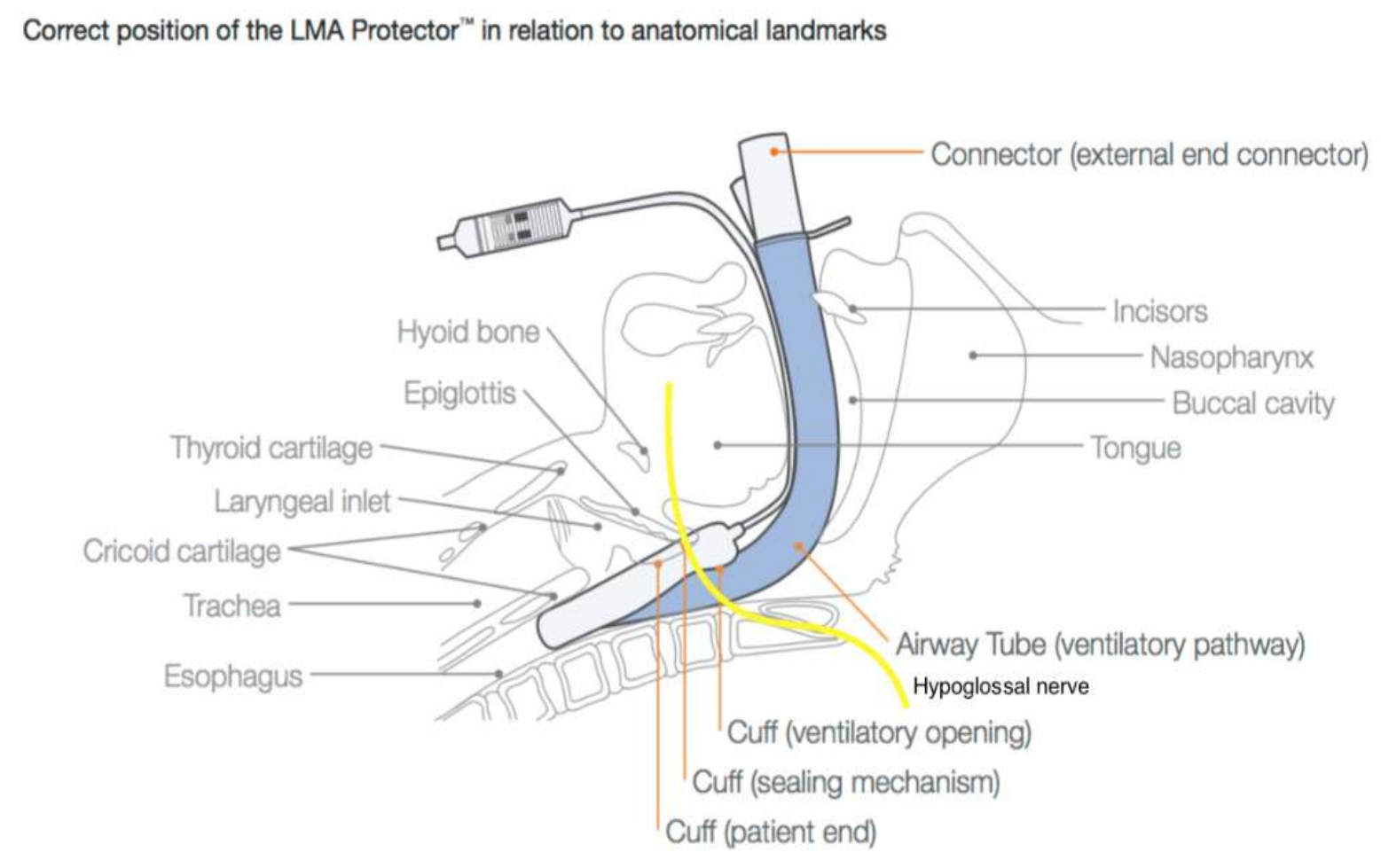

Learning points: In our knowledge, this is the first reported case of unilateral hypoglossal nerve injury after LMA® Protector ${ }^{\mathrm{TM}}$ use. Consideration should be given to down-sizing the device for Asian patients who tend to have smaller jaws for their weight range. We caution that whilst the green zone of the Cuff Pilot ${ }^{\mathrm{TM}}$ corresponds to $40-60 \mathrm{cmH} 2 \mathrm{O}$ cuff pressure, it may be more than the "just-seal" pressure required and the cuff may still be overinflated relative to the size of the patient's airway.

References: 1. Aalap C. Shah. Hypoglossal Nerve Palsy After Airway Management for General Anaesthesia: An Analysis of 69 Patients. Anesth Analg. 2015 Jan; 120(1):105-120 2. Ban Leong Sng et al. A preliminary assessment of the LMA Protector ${ }^{\mathrm{TM}}$ in nonparalysed patients. BMC Anesthesiol. 2017; 17:26

3. A.I.J. Brain. Course of the hypoglossal nerve in relation to the position of the laryngeal mask airway. Anaesthesia, 1995, Volume 50, pages 82-98

4. K. Takahoko et al. Unilateral Hypoglossal Nerve Palsy after Use of the Laryngeal Mask Airway Supreme. Hindawi, Case reports in Anaesthesiology. Volume 2014, Article ID 369563 